\title{
Nanoparticle Complex
}

National Cancer Institute

\section{Source}

National Cancer Institute. Nanoparticle Complex. NCI Thesaurus. Code C62364.

A nanoparticle bound to another moiety of interest via a chemical or physical interaction. 